\title{
Cost Effectiveness of Different Initial Antimicrobial Regimens for Elderly Community-Acquired Pneumonia Patients in General Ward
}

Xiudi Han,' Liang Chen, (D) ${ }^{2}$

Yimin Wang, ${ }^{3}$ Hui Li, ${ }^{3}$ Hong Wang, ${ }^{4}$

Xiqian Xing, ${ }^{5}$ Chunxiao Zhang, ${ }^{6}$

Lijun Suo, ${ }^{7}$ jinxiang Wang, (iD) ${ }^{8}$

Guohua Yu, ${ }^{9}$ Guangqiang Wang, ${ }^{10}$

Xuexin Yao, ${ }^{1}$ Hongxia Yu, ${ }^{12}$ Lei Wang, ${ }^{13}$

Xuedong Liu,' Bin Cao ${ }^{3}$

On behalf of the CAP-China Network

'Department of Pulmonary and Critical Care Medicine, Qingdao Municipal Hospital Group, Qingdao City, Shandong Province, People's Republic of China; ${ }^{2}$ Department of

Infectious Disease, Beijing Jishuitan Hospital, Beijing, People's Republic of China; ${ }^{3}$ National Clinical Research Center of Respiratory Diseases, Center for Respiratory Diseases, Respiratory Diseases, Center for Respiratory Diseases,
China-japan Friendship Hospital, Department of Pulmonary and Critical Care Medicine, China-Japan Friendship Hospital, Beijing, People's Republic of China; ${ }^{4}$ Department of Nosocomial Infection, Qingdao Municipal Hospital Group, Qingdao City, Shandong Province, People's Republic of China: ${ }^{5}$ Department of Pulmonary and Critical Care Medicine, Yan'an Hospital Affiliated to Kunming Medical University, Kunming City, Yunnan Province, People's University, Kunming City, Yunnan Province, People's
Republic of China; 'Department of Pulmonary and Critica Republic of China; 'Department of Pulmonary and Crit'
Care Medicine, Beijing Huimin Hospital, Beijing, People's Republic of China; 'Department of Pulmonary and Critical Care Medicine, Zibo Municipal Hospital, Zibo City, Shandong Province, People's Republic of China; ${ }^{8}$ Department of Pulmonary and Critical Care Medicine, Beijing Luhe Hospital Copit Medical Univerity Beiing People's Republic of Chin Capital Medical University, Bejling, People's Republic of Chin Department of Pulmonary and Critical Care Medicine, Weifang No. 2 People's Hospital, Weifang City, Shandon Province, People's Republic of China; ${ }^{10}$ Department of Respiratory Medicine, Shandong University Affliated Qilu Hospital (Qingdao), Qingdao City, Shandong Province, People's Republic of China; "Department of Respiratory Medicine, The 2nd Hospital of Beijing Corps, Chinese Armed Police Forces, Beiing. Peopl's Republic of Ching. ${ }^{12} \mathrm{Department}$ of Infectio Being, People's Republic of China; "Department of Infectious Disease, Qingdao University Medical College Affliated Yantai Yuhuangding Hospital, Yantai City, Shandong Province, People's Republic of China; ${ }^{13}$ Department of Pulmonary and Critical Care Medicine, Rizhao Chinese Medical Hospital Affliated to Shandong Chinese Medical University, Rizhao City, Shandong Province, People's Republic of Chin

Correspondence: Xuedong Liu

Department of Pulmonary and Critical Care Medicine,

Qingdao Municipal Hospital Group, Jiaozhou Road,

Qingdao City, 2660I I, Shandong Province, People's

Republic of China

$\mathrm{Tel}+86-18661678256$

$\mathrm{Fax}+86-532-82789055$

Email xuedongliu@263.net

Bin $\mathrm{Cao}$

National Clinical Research Center of Respiratory Diseases,

Center for Respiratory Diseases, China-Japan Friendship

Hospital, Department of Pulmonary and Critical Care

Medicine, China-Japan Friendship Hospital, Yinghuayuan

East Street, Chao-yang District, Beijing, 100020, People's

Republic of China

Tel +86-|39| |318339

Fax +86-10-84206264

Email caobin ben@163.com
Purpose: The cost-effectiveness of different guideline-concordant antimicrobial regimens for elderly patients with community-acquired pneumonia (CAP) was rarely discussed. This study attempts to explore the most appropriate cost-effectiveness of guideline-concordant antimicrobial regimen for elderly patients with CAP in general wards.

Patients and Methods: This was a multicenter, retrospective, 4:2:1 matched study enrolling 511 elderly patients with CAP hospitalized in general wards. Two hundred ninety-two patients prescribed with $\beta$-lactam monotherapy (group A), 146 patients prescribed with fluoroquinolone monotherapy (group B) and 73 patients prescribed with $\beta$-lactam/macrolide combination therapy (group C). Clinical outcomes and medical costs were analyzed by $\chi^{2}$ test for categorical variables or Kruskal-Wallis $H$-test for continuous variables.

Results: There were no statistical differences in imaging features, etiology and complications during hospitalization among these three groups. The rates of clinical failure occurrence, in-hospital mortality, 30-day mortality and 60-day mortality also had no significant differences among group A, B and C patients; however, the median length of stay (LOS) in group A patients was 12.0 days, which was significantly higher than that in group B and $\mathrm{C}$ patients (both 10.0 days, $\mathrm{p}<0.02$ ). The median total, drug, and antibiotic costs for one elderly CAP episode in group B patients were RMB 10368.4, RMB 3874.8, and RMB 1796.3, respectively, which were significantly lower than those in group A and C patients $(\mathrm{p}<0.01)$.

Conclusion: Non-inferiority of clinical failure occurrence and short-term mortality was observed in different guideline-concordant antimicrobial regimens for elderly patients with CAP in general wards; however, the median LOS and hospitalization-associated costs for one elderly CAP episode with fluoroquinolone monotherapy were significantly lowest, and this strategy was considered to be the most cost-effective strategy in general wards.

Keywords: community-acquired pneumonia, cost-effectiveness, antimicrobial regimen, elderly, general ward

\section{Introduction}

Community-acquired pneumonia (CAP) is a major cause of morbidity and mortality in adults worldwide. Previous studies showed that one-third of patients with CAP were hospitalized; moreover, hospitalized patients account for over $95 \%$ of the cost of care for CAP. ${ }^{1,2}$ How to reduce CAP-associated costs becomes an important event for government and clinicians. The decreased cost of care of patients with CAP is associated with three aspects. First, the proportion of hospitalized patients with CAP is critical. The 
Infectious Diseases Society of America/American Thoracic Society, ${ }^{3}$ European, ${ }^{4,5}$ and Asian CAP guidelines ${ }^{6,7}$ emphasize the evaluation of pneumonia severity index (PSI) scores $^{8}$ or CURB-65 scores $^{9}$ in patients with CAP. Patients in the lowest risk classes (PSI scores $\leq 70$ or CURB-65 scores $\leq 1$ ) are considered for management in the outpatient setting with hospitalization reserved for those in the higher risk classes (PSI scores $\geq 71$ or CURB-65 scores $\geq 2$ ). Decreasing hospital admissions for CAP is one way to decrease the costs of care. However, in the real world, some patients in low-risk classes, especially those aged $\geq 65$ years, are hospitalized due to social need. ${ }^{10}$ Furthermore, guideline-concordant antimicrobial regimens improve clinical outcomes and decrease length of stay (LOS) and healthcare costs. ${ }^{11-13}$ Concordance to antimicrobial guidelines is associated with lower healthcare costs, and is the most cost-effective strategy for elderly patients with CAP in general wards. ${ }^{13}$ Discordant therapy is an independent risk factor for clinical failure and leads to significantly higher treatment costs than concordant therapy. ${ }^{14}$ Additionally, to a certain extent, an early switch from intravenous antimicrobials to oral therapy, early discharge, and the use of critical pathways can also decrease hospitalization-related costs. ${ }^{15}$

Upon admission, the use of $\beta$-lactam monotherapy, $\beta$-lactam/macrolide combination therapy, and fluoroquinolone monotherapy are recommended as the initial empirical treatment strategies according to CAP guidelines for elderly patients in general wards. ${ }^{3,6}$ Most previous studies discovered that patients prescribed with $\beta$-lactam/macrolide combination therapy and fluoroquinolone monotherapy had similar clinical outcomes $^{15-20}$; however, there were inconsistencies as a few incidences of clinical failure and higher mortality were noted in patients prescribed with fluoroquinolone monotherapy ${ }^{20}$ in those prescribed with $\beta$-lactam monotherapy, respectively. ${ }^{21}$ In practice, apart from providing medical care with good clinical outcomes, clinicians should also consider reducing medical costs. Currently, national healthcare systems pay increasing attention to ccost-effectiveness and ecological effects of antibiotics.

Hence, it is important to know which strategy is the optimal antibiotic treatment strategy among these guidelineconcordant choices. To date, data on the cost-effectiveness of different antimicrobial regimens has rarely been discussed, especially for elderly patients with CAP in general wards. This study attempts to explore the most appropriate costeffective strategy among different guideline-concordant antimicrobial regimens for elderly patients with CAP in general wards.

\section{Materials and Methods}

\section{Study Setting, Design, and Participants}

We conducted a multicenter, retrospective study on hospitalized patients with CAP aged 65 years or older; data were extracted from the CAP-China network. Data of patients that met the inclusion and exclusion criteria were collected from 13 centers in seven cities in three provinces between January 1, 2014, and December 31, 2014 (details are made available in the study by Han et $\mathrm{al}^{10}$ ). The study was approved by the Human Subject Protection Program Institutional Review Board at China-Japan Friendship Hospital. Additional approval obtained from the local institutional review board of each participating hospital. Patient consent was waived owing to the retrospective study design. The initial antimicrobial regimen for each patient was evaluated and categorized as concordance, overtreatment, or undertreatment according to the 2016 Chinese CAP guidelines as previously described. ${ }^{6}$ Guideline-concordant patients admitted to the general ward constituted the main research population in this study. The following treatment regimens are recommended for patients with CAP aged $\geq 65$ years who require non-ICU hospitalization: 1) penicillin/ $\beta$-lactamase-inhibitor combinations; 2) third-generation cephalosporins or their enzyme inhibitor combinations, cephamycins, oxycephalosporins, carbapenems such as ertapenem; 3) monotherapy of the above drugs or in combination with macrolides; and 4) respiratory quinolones. All the detailed antimicrobial regimes are available from supplementary material (Table S1).

\section{Cost Data}

Total cost referred to the sum of all expenditures during hospitalization, including the costs of drugs, nursing, laboratory tests, medical service, and hospitalization, etc. Drug cost referred to the total expenditures on prescribed drugs during hospitalization. Antibiotic cost referred to the total expenditures on prescribed intravenous or oral antibiotics during hospitalization. All costs were calculated using RenMingBi (RMB). The exchange rate of RMB to the United States dollars (USD) at the time of this study was approximately 1:0.16.

Cost-effectiveness was evaluated by the relationship of the total cost, drug cost, antibiotic cost, and outcomes (rate of clinical failure [details are made available in the study by Han et $\mathrm{al}^{14}$ ], mortality, and LOS) among three groups as subsequently defined. The most cost-effective treatment was considered as the empirical antimicrobial regimen with the least cost and the best clinical outcomes. 


\section{Statistical Analysis}

Patients were divided into $\beta$-lactam monotherapy (group A), fluoroquinolone monotherapy (group B), and $\beta$-lactam /macrolide combination therapy (group C). Categorical variables are presented as $\mathrm{n}(\%)$, and continuous variables are presented as mean \pm standard deviation for normally distributed data or median (interquartile range, IQR) for non-normally distributed data. The $\chi^{2}$ test was used for categorical variables, and the analysis of variance test or Kruskal-Wallis $H$-test was used for continuous variables. In order to minimize the selection bias, 4:2:1 paired matching was performed to adjust for differences in baseline characteristics and CAP severity on admission among groups $\mathrm{A}, \mathrm{B}$, and $\mathrm{C}$ patients. We adjusted for confounders of the following characteristics: age, sex, comorbidities (including chronic respiratory disease, cardiovascular disease, chronic liver disease, chronic renal disease, diabetes mellitus, and cerebrovascular disease), risks of aspiration, long-term bedridden status, and PSI scores at admission.

A two-sided $\alpha$ level less than 0.05 was considered statistically significant. Statistical analysis was performed using SPSS software (version 20, IBM Corp., Armonk, NY, USA).

\section{Results}

\section{Patient Characteristics}

After 4:2:1 paired matching, 511 patients aged $\geq 65$ years were enrolled in the final analysis. The screening process is illustrated in Figure 1. There were 292, 146, and 73 patients in groups $\mathrm{A}, \mathrm{B}$, and $\mathrm{C}$, respectively. Patient demographic and clinical characteristics are shown in Table 1 . There were no significant statistical differences in median age, sex, comorbidities, PSI scores, risks of aspiration, and long-term bedridden confinement among the three groups. The number of comorbidities in group B was significantly lower than that in the other two groups $(\mathrm{p}=0.011)$. The proportions of patients with healthcare-associated pneumonia (HCAP) and with risk factors for Pseudomonas aeruginosa (PA) infection in group A were significantly higher than those in the other two groups $(\mathrm{p}<0.001)$.

The presence of the most common symptoms and physiologic abnormalities showed almost no statistical differences, barring expectoration and cyanosis, which were more common in group A than in group B patients. The proportion of patients with lymphocytopenia and $\mathrm{PaO}_{2} / \mathrm{FiO}_{2}<250 \mathrm{mmHg}$ was highest in group A (Table 2).

\section{Radiologic Findings and Complications During Hospitalization}

There were no statistical differences in the presence of imaging features of multilobar infiltration and pleural effusion (Table 2). During the entire hospitalization period, the incidences of complications such as respiratory failure, acute cardiovascular events, and acute renal failure were not statistically different among the groups.

\section{Etiology}

Pathogens were isolated from $12.7 \%$ of the patients $(n=65)$, mainly gram-negative bacteria. Table 3 shows that PA was the most common pathogen in each group, and no atypical pathogens were isolated. There were no statistically significant differences in the presence of common pathogens among the three groups.

\section{Outcomes}

There were no significant differences in the rates of clinical failure occurrence, in-hospital mortality, 30-day mortality, and 60-day mortality among the three groups; however, the median LOS in group A was 12.0 days, which was significantly higher than that in groups $\mathrm{B}$ and C (both 10 days, $\mathrm{p}<0.02$ ) (Figure 2).

The median total, drug, and antibiotic costs for one elderly CAP episode in group B were RMB 10368.4, RMB 3874.8, and RMB 1796.3, respectively, which were lower than those in groups $\mathrm{A}$ and $\mathrm{C}$ patients $(\mathrm{p}<0.01)$ (Figure 3).

\section{Discussion}

To our knowledge, this is the first study to explore the cost-effectiveness of empirical guideline-concordant antimicrobial strategies ( $\beta$-lactam monotherapy, fluoroquinolone monotherapy, and $\beta$-lactam/macrolide combination therapy) in the treatment of hospitalized elderly patients with CAP in China. There was non-inferiority of clinical failure occurrence and short-term mortality among patients who received one of these three treatment regimens. However, patients who received fluoroquinolone monotherapy showed superiority in the median LOS and hospitalization-associated costs than those who received the other two options. Therefore, we considered fluoroquinolone monotherapy to be the most cost-effective strategy for elderly patients with CAP in general wards.

CAP microbiology varies significantly among the elderly in different countries and regions. Previous studies showed 


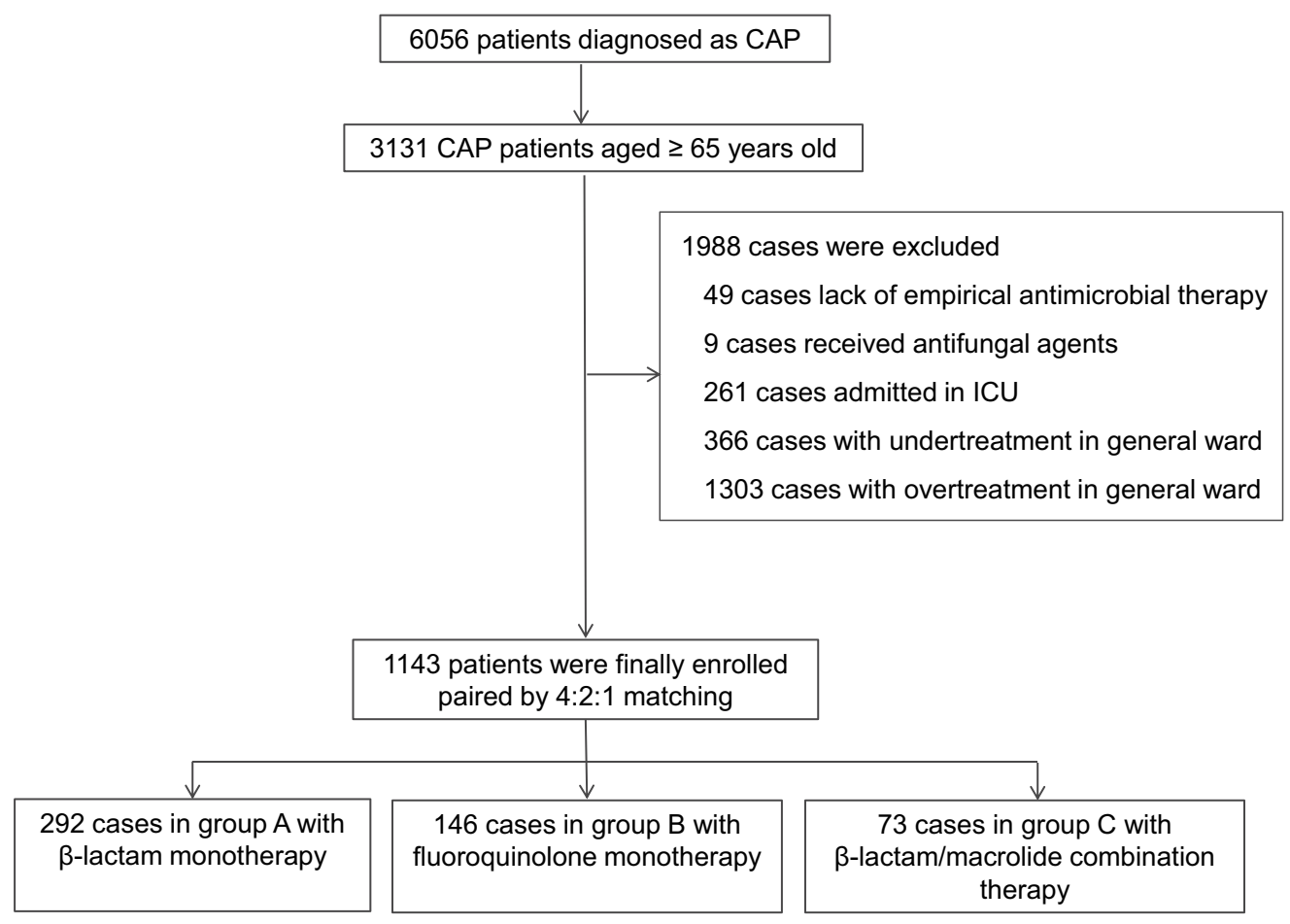

Figure I Screening flow chart of the study.

that the microbiological diagnosis in CAP decreased with increasing age; nonetheless, Streptococcus pneumoniae was still the most frequent pathogen. ${ }^{22,23}$ Isolations of Haemophilus influenzae and multidrug-resistant pathogens were frequent in patients with one or more comorbidities. In our study, approximately $92 \%$ of elderly patients had at least one comorbidity. Based on our data from CAP-China network, gram-negative bacilli, such as PA, were the most common pathogens in elderly patients with CAP. ${ }^{10}$ Appropriate guideline-concordant antimicrobial regimens were prescribed for such patients after an evaluation of risk factors for PA infection. The rate of atypical pathogen

Table I Comparison of Baseline Characteristics in Hospitalized Elderly Patients with CAP in Different Treatment Schemes ( $\mathrm{n}=5 \mathrm{I}$ I)

\begin{tabular}{|c|c|c|c|c|}
\hline Characteristics & Group A (n=292) & Group B $(n=\mid 46)$ & Group C $(n=73)$ & $p$ value \\
\hline Male sex & $125(42.8 \%)$ & $69(47.3 \%)$ & $30(41.1 \%)$ & 0.594 \\
\hline Median age (years, IQR) & $78.1(72.0-83.5)$ & $79.0(72.0-83.0)$ & $78.0(72.0-84.0)$ & 0.966 \\
\hline Underlying conditions & 266(91.1\%) & $132(90.4 \%)$ & $72(98.6 \%)$ & 0.075 \\
\hline Chronic respiratory disease & $91(31.2 \%)$ & $33(22.6 \%)$ & $24(32.9 \%)$ & 0.129 \\
\hline Cardiovascular disease & $184(63.0 \%)$ & $86(58.9 \%)$ & $52(71.2 \%)$ & 0.205 \\
\hline Chronic liver disease & $5(1.7 \%)$ & $2(1.4 \%)$ & $\mathrm{I}(\mathrm{I} .4 \%)$ & 0.953 \\
\hline Chronic renal disease & $18(6.2 \%)$ & $6(4.1 \%)$ & $3(4.1 \%)$ & 0.590 \\
\hline Diabetes mellitus & 47 (16.1\%) & 21 (14.4\%) & $13(17.8 \%)$ & 0.795 \\
\hline Cerebrovascular disease & $82(28.1 \%)$ & $35(24.0 \%)$ & $2 \mathrm{I}(28.8 \%)$ & 0.616 \\
\hline Numbers of comorbidities & $2.0 \pm 1.2$ & $1.8 \pm 1.1$ & $2.3 \pm 1.3$ & 0.011 \\
\hline Risk of aspiration & $28(9.6 \%)$ & $10(6.8 \%)$ & $9(\mid 2.3 \%)$ & 0.392 \\
\hline Long-term bedridden status & $34(I 1.6 \%)$ & $10(6.8 \%)$ & $4(5.5 \%)$ & 0.125 \\
\hline History of CAP within I year & $27(9.2 \%)$ & $8(5.5 \%)$ & $10(13.7 \%)$ & 0.119 \\
\hline PSI scores & $79.0(69.3-96.0)$ & $85.0(70.0-102.0)$ & $88.0(73.5-102.0)$ & 0.123 \\
\hline HCAP & $72(24.7 \%)$ & $17(\mid 1.6 \%)$ & $9(\mid 2.3 \%)$ & 0.001 \\
\hline Risk factors of PA infection ${ }^{\#}$ & $110(37.7 \%)$ & $27(18.5 \%)$ & $14(19.2 \%)$ & $<0.001$ \\
\hline
\end{tabular}

Note: ${ }^{\#}$ Details are made available in the study by Han et al. ${ }^{10}$

Abbreviations: PSI, pneumonia severity index; CAP, community-acquired pneumonia; HCAP, healthcare-associated pneumonia. 
Table 2 Comparison of Clinical Manifestations, Laboratory and Imaging Findings in Hospitalized Elderly Patients with CAP in Different Treatment Schemes $(\mathrm{n}=5 \mathrm{II})$

\begin{tabular}{|c|c|c|c|c|}
\hline Characteristics & Group A $(n=292)$ & Group B $(n=\mid 46)$ & Group C (n=73) & $p$ value \\
\hline \multicolumn{5}{|l|}{ Symptoms } \\
\hline Fever & I39(47.6\%) & $70(47.9 \%)$ & $46(63.0 \%)$ & 0.053 \\
\hline Cough & $268(88.4 \%)$ & $130(89.0 \%)$ & $67(91.8 \%)$ & 0.620 \\
\hline Expectoration & $258(86.7 \%)$ & $114(78.1 \%)$ & $60(82.2 \%)$ & 0.016 \\
\hline Chest pain & $20(6.8 \%)$ & $14(9.6 \%)$ & $7(9.6 \%)$ & 0.529 \\
\hline Wheezing & $122(41.8 \%)$ & $61(41.8 \%)$ & $25(34.2 \%)$ & 0.479 \\
\hline Confusion & $22(7.5 \%)$ & II (7.5\%) & $2(2.7 \%)$ & 0.324 \\
\hline Cyanosis & $47(16.1 \%)$ & II (7.5\%) & $8(11.0 \%)$ & 0.036 \\
\hline \multicolumn{5}{|l|}{ Signs } \\
\hline $\mathrm{RR} \geq 24$ beats $/ \mathrm{min}$ & $8(2.7)$ & $7(4.8)$ & $2(2.7)$ & 0.504 \\
\hline $\mathrm{HR} \geq 125$ beats $/ \mathrm{min}$ & $3(1.0)$ & $0(0)$ & $0(0)$ & 0.323 \\
\hline $\mathrm{SBP}<90 \mathrm{mmHg}$ & $2(0.7)$ & $\mathrm{I}(0.7)$ & $0(0)$ & 0.778 \\
\hline $\mathrm{DBP} \leq 60 \mathrm{mmHg}$ & $50(17.1)$ & $16(11.0)$ & $12(16.4)$ & 0.229 \\
\hline \multicolumn{5}{|l|}{ Laboratory findings } \\
\hline$W B C>10000 / \mathrm{mm}^{3}$ & $67(23.8 \%)$ & $38(26.6 \%)$ & $22(30.6 \%)$ & 0.472 \\
\hline Lymphocyte $<1100 / \mathrm{mm}^{3}$ & $1 \mid 4(4 \mid .6 \%)$ & $39(28.5 \%)$ & $24(33.3 \%)$ & 0.027 \\
\hline $\mathrm{HCT}<30 \%$ & $38(\mid 4.0 \%)$ & $13(9.5 \%)$ & $\mathrm{II}(15.3)$ & 0.353 \\
\hline Albumin<35 g/L & $143(52.8)$ & $62(44.3)$ & $4 I(56.9)$ & 0.144 \\
\hline BUN $>7 \mathrm{mmol} / \mathrm{L}$ & $88(32.1)$ & $37(16.4)$ & $19(26.0)$ & 0.376 \\
\hline $\mathrm{Cr}>123.76 \mathrm{umol} / \mathrm{L}$ & $30(10.9 \%)$ & $12(8.6 \%)$ & $5(6.8)$ & 0.510 \\
\hline Glucose $>14 \mathrm{mmol} / \mathrm{L}$ & $7(2.7 \%)$ & $4(3.0)$ & $2(2.9)$ & 0.984 \\
\hline $\mathrm{Na}<130 \mathrm{mmol} / \mathrm{L}$ & $13(4.7)$ & $6(4.3)$ & $5(6.8)$ & 0.693 \\
\hline $\mathrm{PH}<7.35$ & $9(4.6)$ & $3(3.4)$ & $3(5.2)$ & 0.847 \\
\hline $\mathrm{PaO}_{2} / \mathrm{FiO}_{2}<250 \mathrm{mmHg}$ & $58(30.7)$ & $22(25.0)$ & $8(14.0)$ & 0.041 \\
\hline Lactic acid $>2 \mathrm{mmol} / \mathrm{L}$ & $18(18.2)$ & $6(10.0)$ & $5(10.9)$ & 0.275 \\
\hline ESR & $39.0 \pm 28.6$ & $34.0 \pm 25.2$ & $47.8 \pm 27.5$ & 0.009 \\
\hline CRP & $34.8 \pm 82.9$ & $54.7 \pm 197.5$ & $94.8 \pm 168.7$ & 0.014 \\
\hline \multicolumn{5}{|l|}{ Imaging features } \\
\hline Pleural effusion & $75(25.7)$ & $35(24.0)$ & $18(24.7)$ & 0.924 \\
\hline Multilobe infiltration & $|4|(48.3 \%)$ & $66(45.2 \%)$ & $39(53.4 \%)$ & 0.516 \\
\hline
\end{tabular}

Abbreviations: RR, respiratory rate; HR, heart rate; SBP, systolic blood pressure; DBP, diastolic blood pressure; WBC, white blood cell; HCT, hematocrit; BUN, blood urea nitrogen; $\mathrm{Cr}$, creatinine; $\mathrm{Na}$, sodium; $\mathrm{PaO}_{2} / \mathrm{FiO}_{2}$, partial arterial oxygen pressure/fraction of inspired oxygen; ESR, erythrocyte sedimentation rate; CRP, C-reactive protein.

Table 3 Etiology Distribution in Hospitalized Elderly Patients with CAP in Different Treatment Schemes $(n=65)$

\begin{tabular}{|l|c|c|c|c|}
\hline Pathogens & Group A (n=35) & Group B (n= I7) & Group C (n=13) & P value \\
\hline Pseudomonas aeruginosa & $7(10.8)$ & $2(3.1)$ & $5(7.7)$ & $0.20 \mathrm{I}$ \\
Klebsiella pneumoniae & $7(10.8)$ & 0 & $\mathrm{I}(1.5)$ & 0.102 \\
Escherichia coli & $4(6.2)$ & $\mathrm{I}(1.5)$ & $0(0)$ & 0.397 \\
Acinetobacter & $3(4.6)$ & 0 & $2(3.1)$ & $0.28 \mathrm{I}$ \\
Staphylococcus aureus & $6(9.2)$ & $2(3.1)$ & $0(0)$ & 0.274 \\
Streptococcus pneumoniae & $\mathrm{I}(1.5)$ & $\mathrm{I}(\mathrm{I} .5)$ & $\mathrm{I}(\mathrm{I} .5)$ & 0.746 \\
\hline
\end{tabular}

infections was lower in previous data; ${ }^{10,24}$ moreover, in this study, no atypical pathogens were found. Therefore, the benefit of using $\beta$-lactam/macrolide combination therapy to improve the prognosis of elderly patients with CAP hospitalized in general wards is controversial. A prospective cohort study conducted in Japan enrolled 1131 elderly patients with CAP in general wards, and revealed that azithromycin/ $\beta$-lactam combination therapy 


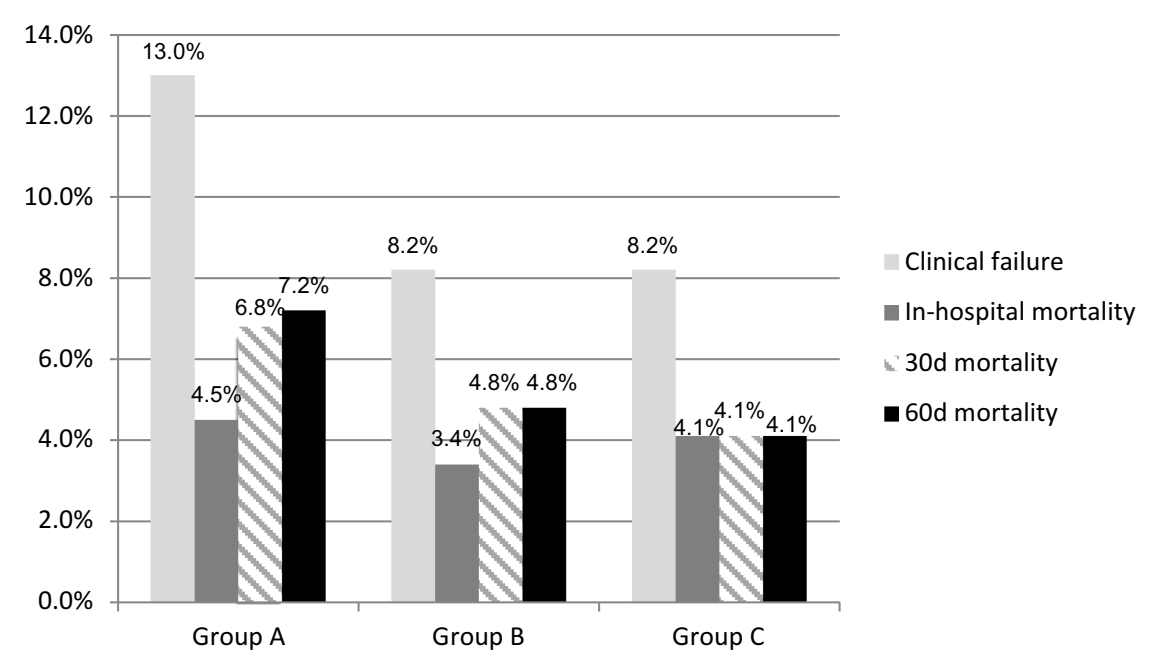

Figure 2 Comparison of rate of clinical failure occurrence, in-hospital mortality, 30-day mortality and 60-day mortality among group A, B and C patients. There were no significant differences among the three groups.

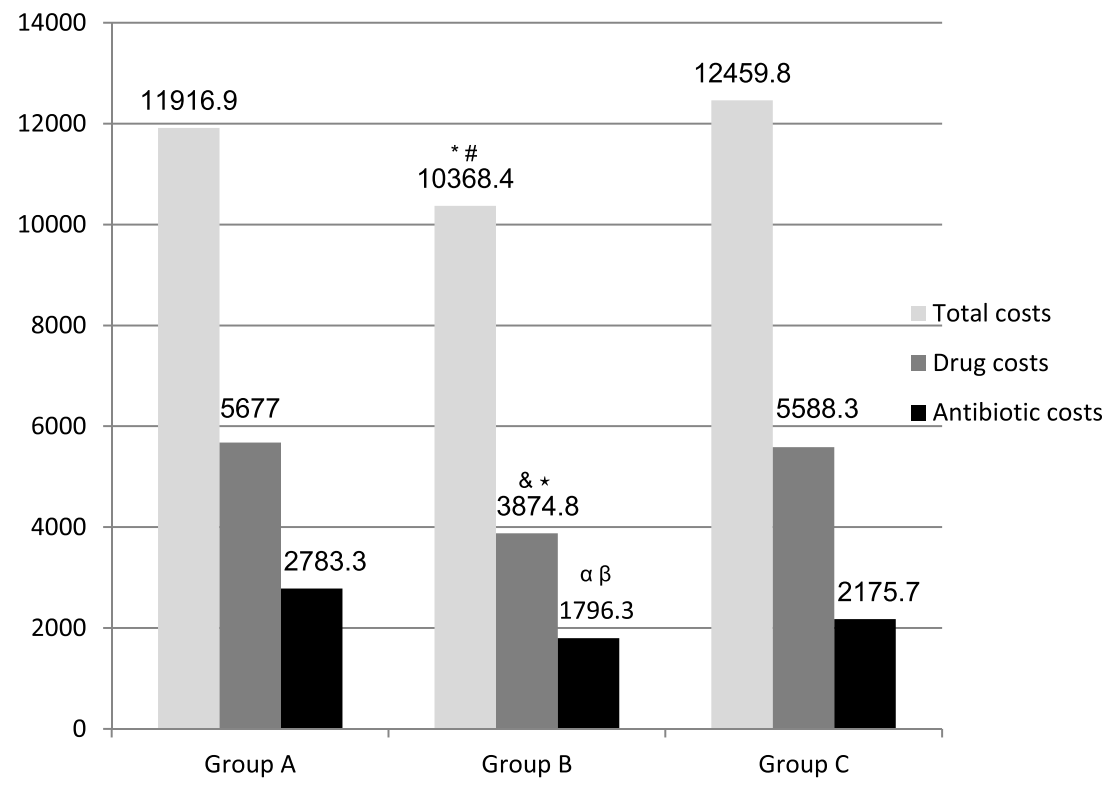

Figure 3 Comparison of the median total costs, drug costs and antibiotic costs for one elderly CAP episode among group A, B and C patients. *Compared with group A of median total costs, $p=0.001$; ${ }^{\#}$ Compared with group $C, p=0.009 ;{ }^{\circledR}$ Compared with group $A$ of median drug costs, $p=0.001 ; *$ Compared with group $C, p=0.009 ;{ }^{\alpha}$ Compared with group $A$ of median antibiotic costs, $p=0.001$; ${ }^{\beta}$ Compared with group $C, p<0.001$.

did not reduce the 30-day mortality compared with that of $\beta$-lactam monotherapy. ${ }^{25}$ This finding was consistent with our results and with the results of a randomized controlled trial (RCT) by Garin et al. ${ }^{26}$ In China, an important characteristic in the etiology of CAP, different from that in most other countries, was a high percentage of $S$. pneumoniae and Mycoplasma pneumoniae infections, which were resistant to macrolides in $88.1-91.3 \%$ and $54.9-60.4 \%$ of the patients, respectively. ${ }^{6}$ Thus, a combination therapy containing macrolides may not provide better advantages in terms of coverage against these two pathogens. A review and metaanalysis including 14 observational studies revealed that adding macrolide to $\beta$-lactams had a favorable effect on mortality only for patients with severe CAP (OR $0.75,95 \%$ CI 0.65-0.86), but not for those with mild to moderate pneumonia (OR 1.12, $95 \%$ CI $0.87-1.45) .{ }^{27}$ Fluoroquinolones have a higher sensitivity to S. pneumoniae and Mycoplasma pneumoniae than macrolides, although our study showed no difference in clinical failure occurrence and mortality with the use of the 
aforementioned three treatment regimens. A systematic review and meta-analysis including nine trials comparing fluoroquinolone monotherapy versus $\beta$-lactam/macrolide combinations demonstrated no difference in mortality; however, the occurrence of clinical failure $(\mathrm{RR}=0.72,95 \% \mathrm{CI}$ $0.57-0.91 ; 2441$ patients) was significantly less common with fluoroquinolone monotherapy. ${ }^{20}$ The inconsistency of the conclusion about clinical failure occurrence may be related to patient age and disease severity. Our study focused only on mild to moderate CAP in elderly patients.

LOS is associated with patient age, disease severity, and treatment strategy. An older patient population, critically ill patients, and the use of guideline-discordant antibiotic options can increase the LOS. An open-label, multicenter, non-inferiority, randomized trial comparing $\beta$-lactam monotherapy with $\beta$-lactam/macrolide combination therapy in 580 moderately severe patients with CAP found that LOS did not differ between the two arms. ${ }^{26}$ A recent meta-analysis of 22 trials involving 6235 non-ICU patients with CAP, conducted by Liu et al, showed that compared with $\beta$-lactam with or without the combination of macrolides, fluoroquinolone monotherapy reduced the median LOS by 0-2 days. However, there was no difference in the mean LOS. ${ }^{28}$ Interestingly, our study findings demonstrated that the median LOS in patients receiving fluoroquinolone monotherapy and $\beta$-lactam/macrolide combination therapy was not statistically different, but significantly lower than that in patients receiving $\beta$-lactam monotherapy (10.0 days versus 12.0 days, $\mathrm{p}<0.01)$. Meanwhile, we found that the upper quartile of the LOS was higher during the fluoroquinolone monotherapy period than during the $\beta$-lactam/macrolide combination therapy period. We considered that the immunomodulatory effect of macrolides on host-pathogen interaction, the functions of epithelial and inflammatory cells, the improvement of mucociliary clearance, and the attenuation of the inflammatory response may play a role in altering the LOS.

The financial implication associated with CAP is a considerable burden to the healthcare systems of different countries. Better prognosis, lower antibacterial therapy costs, and shorter LOS are the most concerned issues for physicians. It is well known that the use of guidelineconcordant antimicrobial therapy in elderly patients with CAP is associated with a significantly shorter LOS, decreased mortality, and reduced costs. ${ }^{10,13}$ For patients with more severe CAP in PSI risk classes IV and V, fluoroquinolone monotherapy ( $94 \%$ survival, cost-effectiveness ratio $[\mathrm{CER}]=4635 \mathrm{USD}$; costs adjusted to 2005) was associated with a more favorable CERs than $\beta$-lactam/macrolide combination therapy (98\% survival, $\mathrm{CER}=5278 \mathrm{USD}){ }^{29}$ However, the most cost-effective initial empirical treatment choice for the elderly in general wards remains unclear, as there is little evidence for clinicians to refer; thus, a formal cost-effectiveness analysis is needed. A cluster-randomized crossover trial involving 2283 patients from the Community-Acquired Pneumonia Study on the initial Treatment with Antibiotics of Lower Respiratory Tract Infections showed that there were no significant differences in CERs of CAP in general wards among the aforementioned three regimens. ${ }^{30}$ This finding was different from our study finding, probably due to differences in selected populations; we focused on patients aged $\geq 65$ years. In our study, patients who received fluoroquinolone monotherapy had significantly lower total, drug, and antibiotic costs than those who received the other two regimens, suggesting that fluoroquinolone monotherapy is a more cost-effective strategy for elderly patients with CAP.

In addition to efficacy and cost, drug safety and the convenience of administration are also important for the elderly. Fluoroquinolones require a once-daily administration; additionally, data from a meta-analysis indicated that the use of fluoroquinolone was associated with significantly fewer adverse events compared with the use of $\beta$-lactam $/$ macrolide combination therapy (RR $0.74,95 \%$ CI $0.610 .90 .^{28}$

Our study has some limitations. First, it was a retrospective pair-matching study; therefore, it could not provide good-quality comparative clinical data. The matching process may have had selection bias. Thus, future prospective RCTs are warranted. Second, the number of enrolled patients was insufficient. Lastly, the socalled initial empiric therapy was not the real initial empiric therapy, as some patients had a documented history of pre-hospital medication.

\section{Conclusion}

This is the first study conducted to assess the costeffectiveness of three empirical guideline-concordant antimicrobial therapeutic strategies for non-ICU elderly patients with CAP in China. Our results demonstrated no significant differences in clinical failure occurrence and short-term mortality among patients receiving one of these three strategies. Nevertheless, LOS and total, drug, and antibiotic costs were significantly lower in patients who received fluoroquinolone monotherapy than in those who received $\beta$ lactam with or without macrolide therapy. Fluoroquinolone monotherapy is considered to be the most cost-effective strategy for elderly patients with CAP in general wards. 


\section{Ethics Approval}

This study was approved by the China-Japan Friendship Hospital Ethics Committee (No. 2015-85) on October 12, 2015. We also confirmed that all patient data was treated with confidentiality, in accordance with the Declaration of Helsinki.

\section{Acknowledgments}

The authors are grateful for the contributions of all the staff of the CAP-China network for their help with data collection and input.

\section{Author Contributions}

All authors made a significant contribution to the work reported, whether that is in the conception, study design, execution, acquisition of data, analysis and interpretation, or in all these areas; took part in drafting, revising or critically reviewing the article; gave final approval of the version to be published; have agreed on the journal to which the article has been submitted; and agree to be accountable for all aspects of the work.

\section{Funding}

This work was supported by the National Science Grant for Distinguished Young Scholars (grant number 81425001/H0104), the National Key Technology Support Program from Ministry of Science and Technology (grant number 2015BAI12B11) and the Beijing Science and Technology Project (grant number D151100002115004).

\section{Disclosure}

The authors report no conflicts of interest in this work.

\section{References}

1. Marrie TJ. Community-acquired pneumonia. Clin Infect Dis. 1994;18:501-505. doi:10.1093/clinids/18.4.501

2. Guest JF, Morris A. Community-acquired pneumonia: the annual cost to the National Health Service in the UK. Eur Respir J. 1997;10:1530-1534. doi:10.1183/09031936.97.10071530

3. Metlay JP, Waterer GW, Long AC, et al. Diagnosis and treatment of adults with community-acquired pneumonia. An official clinical practice guideline of the American Thoracic Society and Infectious Diseases Society of America. Am J Respir Crit Care Med. 2019;200 (7):e45-e67. doi:10.1164/rccm.201908-1581ST

4. National Institute for Health and Care Excellence. Pneumonia in adults: diagnosis and management. London, UK: National Institute for Health and Care Excellence; 2019.

5. Wiersinga WJ, Bonten MJ, Boersma WG, et al. Management of community-acquired pneumonia in adults: 2016 guideline update from the Dutch Working Party on Antibiotic Policy (SWAB) and Dutch Association of Chest Physicians (NVALT). Neth J Med. 2018;76(1):4-13.
6. Cao B, Huang Y, She DY, et al. Diagnosis and treatment of community-acquired pneumonia in adults: 2016 clinical practice guidelines by the Chinese Thoracic Society, Chinese Medical Association. Clin Respir J. 2018;12(4):1320-1360. doi:10.1111/crj.12674

7. Lee MS, Oh JY, Kang CI, et al. Guideline for antibiotic use in adults with community-acquired pneumonia. Infect Chemother. 2018;50 (2):160-198. doi:10.3947/ic.2018.50.2.160

8. Fine MJ, Auble TE, Yealy DM, et al. A prediction rule to identify low-risk patients with community acquired pneumonia. $N$ Engl J Med. 1997;336(4):243-250. doi:10.1056/NEJM199701233360402

9. Lim WS, van der Eerden MM, Laing R, et al. Defining community acquired pneumonia severity on presentation to hospital: an international derivation and validation study. Thorax. 2003;58(5):377-382. doi:10.1136/thorax.58.5.377

10. Han X, Zhou F, Li H, et al. Effects of age, comorbidity and adherence to current antimicrobial guidelines on mortality in hospitalized elderly patients with community-acquired pneumonia. BMC Infect Dis. 2018;18(1):192. doi:10.1186/s12879-018-3098-5

11. Arnold FW, LaJoie AS, Brock GN, et al. Improving outcomes in elderly patients with community-acquired pneumonia by adhering to national guidelines: Community-acquired Pneumonia Organization International cohort study results. Arch Intern Med. 2009;169 (16):1515-1524. doi:10.1001/archinternmed.2009.265

12. Frei CR, Restrepo MI, Mortensen EM, Burgess DS. Impact of guideline-concordant empiric antibiotic therapy in community-acquired pneumonia. Am J Med. 2006;119(10):865-871. doi:10.1016/j.amjmed.2006.02.014

13. Egger ME, Myers JA, Arnold FW, Pass LA, Ramirez JA, Brock GN. Cost effectiveness of adherence to IDSA/ATS guidelines in elderly patients hospitalized for Community-Acquired Pneumonia. BMC Med Inform DecisMak. 2016;16:34. doi:10.1186/s12911-016-0270-y

14. Han X, Liu X, Chen L, et al. CAP-China network. Disease burden and prognostic factors for clinical failure in elderly community acquired pneumonia patients. BMC Infect Dis. 2020;20(1):668. doi:10.1186/s12879-020-05362-3

15. Marrie TJ, Lau CY, Wheeler SL, Wong CJ, Vandervoort MK, Feagan BG. A controlled trial of a critical pathway for treatment of community-acquired pneumonia. CAPITAL study investigators. Community-acquired pneumonia intervention trial assessing levofloxacin. JAMA. 2000;283(6):749-755. doi:10.1001/ jama.283.6.749

16. Lee JH, Kim SW, Kim JH, Ryu YJ, Chang JH. High-dose levofloxacin in community-acquired pneumonia: a randomized, open-label study. Clin Drug Investing. 2012;32:569-576. doi:10.1007/ BF03261911

17. Lin TY, Lin SM, Chen HC, et al. An open-label, randomized comparison of levofloxacin and amoxicillin/clavulanate plus clarithromycin for the treatment of hospitalized patients with community-acquired pneumonia. Chang Gung Med J. 2007;30:321-332.

18. Portier H, Brambilla C, Garre M, Paganin F, Poubeau P, Zuck P. Moxifloxacin monotherapy compared to amoxicillin-clavulanate plus roxithromycin for nonsevere community-acquired pneumonia in adults with risk factors. Eur $J$ Clin Microbiol Infect Dis. 2005;24:367-376. doi:10.1007/s10096-005-1347-1

19. Postma DF, van Werkhoven $\mathrm{CH}$, van Elden LJ, et al. Antibiotic treatment strategies for community-acquired pneumonia in adults. N Engl J Med. 2015;372:1312-1323. doi:10.1056/NEJMoa1406330

20. Raz-Pasteur A, Shasha D, Paul M. Fluoroquinolones or macrolides alone versus combined with b-lactams for adults with community acquired pneumonia: systematic review and meta-analysis. Int $J$ Antimicrob Agents. 2015;46:242-248. doi:10.1016/j.ijantimicag.2015.04.010

21. Lee JS, Giesler DL, Gellad WF, Fine MJ. Antibiotic therapy for adults hospitalized with community-acquired pneumonia: a systematic review. JAMA. 2016;315:593-602. doi:10.1001/jama. 2016.0115 
22. Vila-Corcoles A, Ochoa-Gondar O, Rodriguez-Blanco T, Raga-Luria X, Gomez-Bertomeu F. Epidemiology of community-acquired pneumonia in older adults: a population-based study. Respir Med. 2009;103(2):309-316. doi:10.1016/j.rmed.2008.08.006

23. Cillóniz C, Polverino E, Ewig S, et al. Impact of age and comorbidity on cause and outcome in community-acquired pneumonia. Chest. 2013;144:999-1007. doi:10.1378/chest.13-0062

24. Fung HB, Monteagudo-Chu MO. Community-acquired pneumonia in the elderly. Am J Geriatr Pharmacother. 2010;8:47-62. doi:10.1016/ j.amjopharm.2010.01.003

25. Ito A, Ishida T, Tachibana H, Tokumasu H, Yamazaki A, Washio Y. Azithromycin combination therapy for community-acquired pneumonia: propensity score analysis. Sci Rep. 2019;9(1):18406. doi:10.1038/s41598-019-54922-4

26. Garin N, Genné D, Carballo S, et al. $\beta$-Lactam monotherapy vs. $\beta$ lactam-macrolide combination treatment in moderately severe community-acquired pneumonia: a randomized noninferiority trial. JAMA Intern Med. 2014;174(12):1894-1901. doi:10.1001/jamainternmed. 2014.4887
27. Horita N, Otsuka T, Haranaga S, et al. Beta-lactam plus macrolides or beta-lactam alone for community-acquired pneumonia: a systematic review and meta-analysis. Respirology. 2016;21(7):1193-1200. doi:10.1111/resp. 12835

28. Liu S, Tong X, Ma Y, et al. Respiratory fluoroquinolones monotherapy vs. beta-lactams with or without macrolides for hospitalized community-acquired pneumonia patients: a meta-analysis. Front Pharmacol. 2019;10:489. doi:10.3389/fphar.2019.00489

29. Frei CR, Burgess DS. Cost-effectiveness of four antimicrobial regimens in patients admitted to the medical floor with class IV or V community-acquired pneumonia. Value Health. 2004;7(3):355. doi:10.1016/S1098-3015(10)62484-9

30. Van Werkhoven $\mathrm{CH}$, Postma DF, Mangen MJ, Oosterheert JJ, Bonten MJ. Cost-effectiveness of antibiotic treatment strategies for community-acquired pneumonia: results from a cluster randomized cross-over trial. BMC Infect Dis. 2017;17(1):52. doi:10.1186/s12879016-2179-6
Infection and Drug Resistance

\section{Publish your work in this journal}

Infection and Drug Resistance is an international, peer-reviewed openaccess journal that focuses on the optimal treatment of infection (bacterial, fungal and viral) and the development and institution of preventive strategies to minimize the development and spread of resistance. The journal is specifically concerned with the epidemiology of
Dovepress

antibiotic resistance and the mechanisms of resistance development and diffusion in both hospitals and the community. The manuscript management system is completely online and includes a very quick and fair peerreview system, which is all easy to use. Visit http://www.dovepress.com/ testimonials.php to read real quotes from published authors. 